\title{
Strain relaxation and segregation effects during self-assembled InAs quantum dots formation on $\mathrm{GaAs}(001)$
}

\author{
J. M. García, ${ }^{\text {a) }}$ J. P. Silveira, and F. Briones \\ Instituto de Microelectrónica de Madrid, CNM-CSIC, C./Isaac Newton, 8, Tres Cantos, \\ 28760-Madrid, Spain
}

(Received 29 November 1999; accepted for publication 30 May 2000)

\begin{abstract}
In segregation effects during InAs growth on $\mathrm{GaAs}(001)$ and critical thickness for InAs self-assembled quantum dots are studied using a real time, in situ technique capable of measuring accumulated stress during growth. Due to a large $(\sim 50 \%)$ surface In segregation of floating In, self-assembled dot formation takes place when less than one monolayer of InAs is pseudomorphically grown on GaAs. A picture of the growth process is discussed on the basis of the equilibrium between InAs and floating In dominated by the stress energy. (C) 2000 American Institute of Physics. [S0003-6951(00)05529-7]
\end{abstract}

Growth kinetics effects, critical thickness, and heteroepitaxial strain consideration are decisive for knowing how to build high quality self-assembled quantum dots (QD) structures. Coherent InAs island formation on GaAs(001), induced by elastic strain partial relaxation, can provide a practical growth approach to the fabrication of optically efficient QD devices as lasers ${ }^{1}$ or QD infrared photodetectors. ${ }^{2}$ Moreover, QD have been used as a powerful experimental tool to test basic theories on low dimensional systems.

It is well known that during heteroepitaxial growth of III-V materials, segregation of column III elements ${ }^{3}$ is a serious problem for growing high quality interfaces. Many works have focussed on In segregation effects during depositions below the critical thickness for QD formation using both ex situ ${ }^{4-6}$ and in situ techniques. Although some methods ${ }^{8}$ have been proposed for improving InAs quantum well structures, dealing with segregation on QD is a much more complex problem. In particular, indium segregation effects in QD can lead also to important changes of shape and composition, ${ }^{9}$ influencing the energy levels of the confined states. ${ }^{10} \mathrm{~A}$ precise understanding of this process can be used to control the size and the shape of QD. ${ }^{9}$

This letter presents a study of strain relaxation and In segregation effects during InAs growth on $\mathrm{GaAs}(001)$ both below and above critical thickness for QD formation. We have used a technique capable of quantify accumulated stress measuring the deflection of a laser beam by the cantilevershaped sample. Stress relaxation due to QD formation is observed and will be discussed. We propose a more complex growth mode of InAs on GaAs in which stress energy induces In surface segregation.

In situ, real time measurements of accumulated stress during heteroepitaxial molecular-beam epitaxy (MBE) growth are performed by direct determination of strain induced substrate curvature using a laser deflection technique. ${ }^{11,12}$ Cantilever-shaped $\mathrm{GaAs}(001)$ substrates, cut along [110] and [1 $1 \overline{0}]$ directions, are mechanically polished to $80-100 \mu \mathrm{m}$ thickness, mounted on a special substrate holder that let the sample bend, and directly heated by radia-

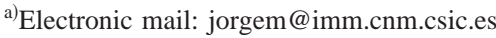

tion from a filament. Substrate temperature $\left(T_{s}\right)$ is carefully calibrated by determining oxide desorption temperature and surface reconstruction phase transitions by means of reflection high-energy electron diffraction (RHEED). Accumulated stress, by definition the stress-thickness product integrated along the grown layers ( $\mathrm{N} / \mathrm{m}$ units), can be quantitatively determined for thin film thickness measuring cantilever bending. We have used Stoney's equation, ${ }^{13}$ that relates sample radius of curvature with substrate elastic constants and set-up geometry. Although a QD assembly will induce a nonuniform spatial distribution of stress, it can be characterized by an effective stress thickness ${ }^{12}$ as for a planar film of equivalent thickness. Our experimental set up is very sensitive, e.g., being able to detect changes in stress produced by less than 0.01 monolayers (ML) of InAs on GaAs(001). No significant thermal drifts are induced by radiation from the Knudsen cells, as can be deduced from the stress signal stability after closing In or Ga cells shutters. During the experiment, the sample is exposed continuously to $2 \times 10^{-6}$ mbar beam equivalent $\mathrm{As}_{4}$ pressure, i.e. both during In deposition and growth pause. InAs growth rate used $(0.036 \mathrm{ML} / \mathrm{s})$ was carefully calibrated using RHEED oscillations during epitaxial growth of thick layers of low In contents $(x<0.08) \operatorname{In}_{x} \mathrm{Ga}_{1-x}$ As on $\mathrm{GaAs}(001)$ where In segregation effect should be negligible.

Two kind of structures have been grown on the cantilevers under real time observation of stress evolution: single monolayer (SM) and QD samples. For SM samples, a dose of In atoms sufficient to grow one nominal monolayer of InAs (1 In ML) $)^{14}$ was supplied at different $T_{s}$ from 200 to $500{ }^{\circ} \mathrm{C}$. Detailed results on SM samples are reported elsewhere. ${ }^{15}$ For QD samples, In is supplied at $T_{s}=500^{\circ} \mathrm{C}$, up to doses slightly higher than the required critical thickness for $\mathrm{QD}$ formation $\left(\Theta_{\mathrm{QD}}\right)$. RHEED technique was used to monitor the two-dimensional (2D) to three-dimensional (3D) transition. Subsequent GaAs capping layer growth (0.593 $\mathrm{ML} / \mathrm{s}$ ) was initiated at $500^{\circ} \mathrm{C}$ during deposition of the first $10 \mathrm{~nm}$ and then $T_{s}$ was ramped to $585^{\circ} \mathrm{C}$ in order to obtain high quality GaAs.

In all studied SM samples, during deposition of 1 equivalent ML of In, after a brief initial transient, probably 


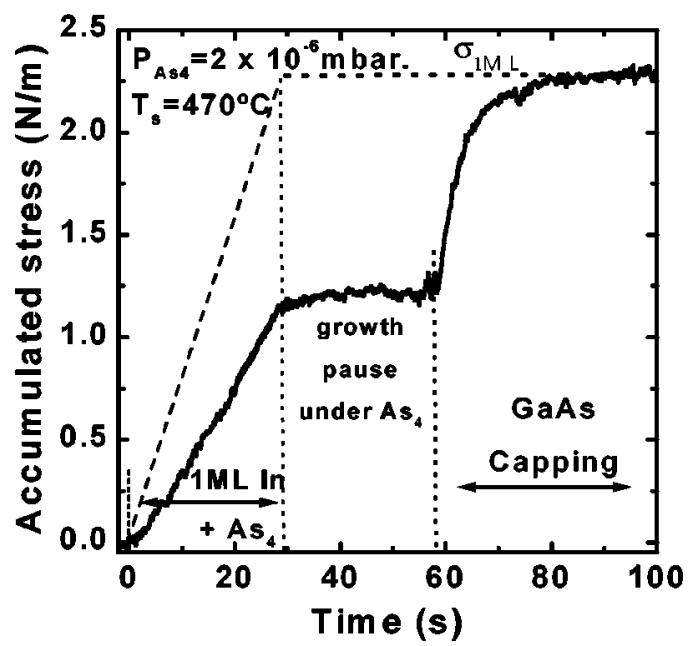

FIG. 1. Accumulated stress evolution during 1 In $\mathrm{ML}+\mathrm{As}_{4}$ deposition, growth interruption, and subsequent GaAs capping. Total accumulated stress is $\sim 2.25 \mathrm{~N} / \mathrm{m}$. Straight line corresponds to the ideal accumulated stress increase rate if all supplied In were incorporating as InAs.

related to surface reconstruction changes, ${ }^{11}$ an approximately linear increase of accumulated stress is observed. Except for this initial transient behavior, observed stress evolution is isotropic, identical for [110] and [1 10$]$ cut cantilevers, as expected for an isotropic mismatched layer. Figure 1 shows accumulated stress evolution at $T_{s}=470{ }^{\circ} \mathrm{C}$. The figures presented in this work are [110] samples. A flat plateau is observed during a subsequent $30 \mathrm{~s}$ growth interruption. Consequently, we do not expect any In desorption from the surface at this growth temperature. ${ }^{16}$

Furthermore, during capping of InAs with GaAs, we observe again a progressive increase of accumulated stress until a final steady stress state $\left(\sigma_{1 \mathrm{ML}}\right)$ is reached for a GaAs layer thickness below $10 \mathrm{~nm}$ for all the studied growth conditions. Interpretation of this observation is clear if we consider that our experimental method is only sensitive to the fraction of In that induces stress: during deposition of In, only a fraction of the delivered In is incorporated as InAs. The rest accumulates (or floats) on the surface contributing insignificantly to the increase of stress. We want to point out that although both In and As have been supplied together, the actual growth rate of pseudomorphic InAs based in our results is approximately half of the ideal one (dashed line, Figure 1). Therefore, we prefer to address the amount of delivered material as In and not InAs. During subsequent GaAs capping deposition, this floating In is progressively incorporated together with GaAs and increases stress until total In exhaustion. The fraction of floating In can be evaluated from the ratio between partial accumulated stress reached during InAs deposition and total accumulated stress induced after capping with a thick GaAs layer. At this point, total accumulated stress reaches a value of $\sim 2.25 \mathrm{~N} / \mathrm{m}$. For the studied growth temperatures ${ }^{15}$ (from 200 to $500{ }^{\circ} \mathrm{C}$ ) $\sigma_{1 \mathrm{ML}}$ is 2.2 $\pm 0.2 \mathrm{~N} / \mathrm{m}$. Also we find that for the growth temperatures commonly used $\left(T_{s}>450{ }^{\circ} \mathrm{C}\right)$ for QD growth $50 \%$ of the supplied In does not incorporate actually into the InAs wetting layer (WL). ${ }^{16}$ It only incorporates progressively later during GaAs capping growth. This process shows that In segregation is not controlled only by a surface exchange process between III column atoms, as previously suggested, ${ }^{3}$ but Downloaded 26 Feb 2010 to 161.111.180.191. Redistribution subject

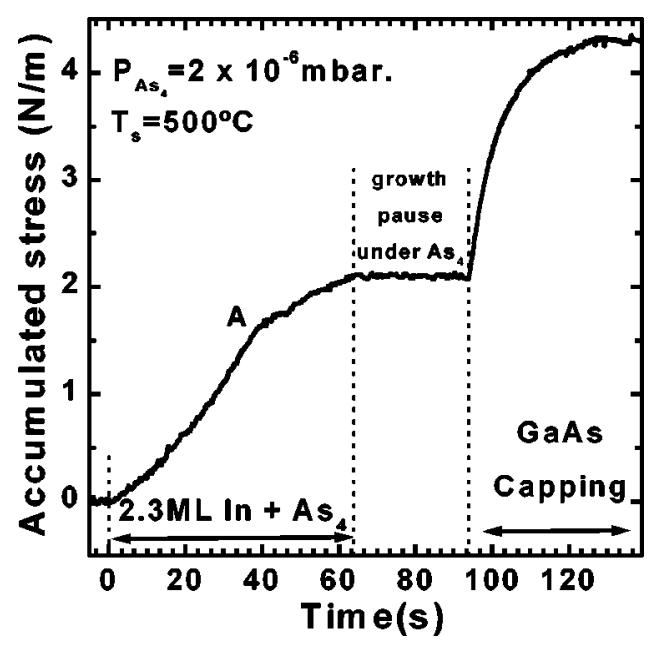

FIG. 2. Accumulated stress evolution during 2.3 In $\mathrm{ML}+\mathrm{As}_{4}$ deposition, growth interruption, and subsequent GaAs capping. A clear reduction of stress increase rate is observed at point A due to QD formation, in coincidence with the appearance of a 3D RHEED pattern.

mainly by surface stress effects. Our results demonstrate the importance of strain-driven ${ }^{17}$ kinetics on the surface evolution during the growth of highly mismatched heterostructures. The linear dependence of accumulated stress with time during In supply suggest that the amount of incorporated InAs is proportional and in equilibrium with the In supersaturation on the surface. The segregation profile during GaAs capping is influenced by the progressive incorporation and depletion of this floating In under a combination of both thermodynamic equilibrium and growth kinetic mechanisms.

Using a simple model for the induced plastic deformation, according to Ref. 12, the expected stress introduced for 1 InAs ML coherently incorporated can be calculated as: $\sigma_{1 \mathrm{ML}}=\left(M_{\mathrm{InAs}}\left(a_{\mathrm{InAs}} a_{\mathrm{GaAs}}\right) / a_{\mathrm{InAs}}\right) h_{1 \mathrm{ML}}$, where $M_{\mathrm{InAs}}=\left(c_{11}\right.$ $\left.+c 12-2 c_{12}^{2} / c_{11}\right)$ is the biaxial modulus for an $\operatorname{InAs}(001)$ oriented crystal, $c_{11}$ and $c_{12}$ are InAs bulk elastic modulus, $a_{\mathrm{InAs}}\left(a_{\mathrm{GaAs}}\right)$ is the InAs $(\mathrm{GaAs})$ lattice parameter, and $h_{1 \mathrm{ML}}=0.32 \mathrm{~nm}$ is assumed to be the thickness of one inplane compressed InAs ML film. Using $c_{11}=83.3 \mathrm{GPa}$ and $c_{12}=45.3 \mathrm{GPa},{ }^{18}$ calculated $\sigma_{1 \mathrm{ML}}$ is $3.7 \mathrm{~N} / \mathrm{m}$ per ML. Surprisingly, this value, although bulk elastic constants are used, is in reasonable agreement with our measurements.

The large amount of segregated In strongly modifies the standard picture for QD self-assembling process. Figure 2 shows accumulated stress evolution during continuous supply of $2.3 \mathrm{ML}$ of InAs at $500{ }^{\circ} \mathrm{C}$. A $50 \%$ reduction of stress increase rate is observed at point A corresponding to $\Theta_{\mathrm{QD}}$ $=1.4 \mathrm{ML}$. A 3D RHEED pattern appears approximately at that moment. We have observed, systematically, a critical thickness for QD formation of 1.4-1.6 ML. Although slightly below some reported values, ${ }^{1}$ it is in good agreement with other works ${ }^{17,19} \Theta_{\mathrm{QD}}$ in which $T_{s}$ was closer to ours. Narrow size distribution of QD is confirmed both by atomic force microscopy (AFM) of uncapped samples as well as by narrow low temperature photoluminescence emission at 1.2 $\mathrm{eV}$ of capped samples.

When the QD are formed, the surface must be in a quasiequilibrium of three phases: 2D InAs islands, QD, and floating In. Due to the large lattice mismatch InAs/GaAs, strain energy competes efficiently with chemical bonding energy, to AIP license or copyright; see http://apl.aip.org/apl/copyright.jsp 
which is controlled by surface In supersaturation. This competition determines the equilibrium ratio between bonded (InAs) and unbonded In (floating In). The appearance of QD (with smaller associated stress accumulation due to surface relaxation) displaces previous equilibrium and reduces the observed accumulated stress slope as a function of delivered In (Fig. 2, point A). However, quantitative analysis of stress relaxation due to QD formation is complicated by mass transfer process between rapidly migrating In atoms, InAs 2D islands, and QD. The coexistence of these phases have been observed ${ }^{17}$ at this growth stage by in situ scanning tunneling microscope (STM). QD formation process is still controlled by the amount of supplied In and thermodynamic equilibrium.

The large increase of stress observed during GaAs capping (Fig. 2) is due mainly to the incorporation of floating In, but its final value cannot be directly and quantitatively related to the amount of this In. We should take into account the inhomogeneous nature of the stress field associated with the buried dots. On the other hand, Ga supply during capping layer growth strongly modifies equilibrium between InAs and floating In. This makes it possible for the In to incorporate in the form of an $\mathrm{In}_{x} \mathrm{Ga}_{1-x}$ As alloy with a gradually decreasing $x$ composition. Strong In-Ga intermixing has been identified to be responsible for QD size change during capping. ${ }^{9}$

Two relevant outcomes can be derived from previous results. First, consequence is that the real critical thickness for $2 \mathrm{D} / 3 \mathrm{D}$ transition is less than $1 \mathrm{ML}$. Reported values in the literature for the critical thickness ranges from 1.4 to 2.0 ML. However, our results show that the actual amount of growing 2D InAs contributing to the accumulated stress must be less than $1 \mathrm{ML}$. Our critical thickness values are in good agreement with some predictions based on elastic energy calculations. ${ }^{20}$ The second outcome is that the InAs wetting layer presents no sharp interface. Instead of a WL, a graded composition InGaAs layer embeds QD.
In conclusion, the simple Stranski-Krastanow model (in which after reaching a critical thickness the 2D grown layer breaks into 3D dots) does not seems to apply to a system where strong segregation effects are present as in the case of InAs on GaAs. A more complex picture arises from our direct stress measurements in which strong stress-induced In surface segregation controls mass transport and growth phenomena responsible for QD self-assembly.

The authors wish to acknowledge D. Gómez for cantilever preparation and R. García for AFM measurements.

${ }^{1}$ N. Kirstaedter, O. G. Schmidt, N. N. Ledentsov, D. Bimberg, V. M. Ustinov, A. Egorov Yu, A. E. Zhukov, M. V. Maximov, P. S. Kop'ev, and Zh. I. Alferov, Appl. Phys. Lett. 69, 1226 (1996).

${ }^{2}$ S. Maimon, E. Finkman, G. Bahir, S. E. Schacham, J. M. García, and P. M. Petroff, Appl. Phys. Lett. 73, 2003 (1998).

${ }^{3}$ J. M. Moison, C. Guille, F. Houzay, F. Barthe, and M. VanRompay, Phys. Rev. B 40, 6149 (1989).

${ }^{4}$ M. Sato and Y. Horikoshi, J. Appl. Phys. 69, 7697 (1991).

${ }^{5}$ K. H. Ploog and O. Brandt, Semicond. Sci. Technol. 8, S229 (1993).

${ }^{6}$ K. Muraki, S. Fukatsu, Yshiraki, and R. Ito, Appl. Phys. Lett. 61, 557 (1992).

${ }^{7}$ J. M. Gerard, Appl. Phys. Lett. 61, 2096 (1992).

${ }^{8}$ E. Tournier, O. Brandt, and K. Ploog, Appl. Phys. Lett. 60, 287 (1992).

${ }^{9}$ J. M. García, G. Medeiros-Ribeiro, K. Schmidt, T. Ngo, and P. M. Petroff, Appl. Phys. Lett. 71, 2014 (1997).

${ }^{10}$ J. M. García, T. Mankand, T. Holtz, P. J. Wellmann, and P. M. Petroff, Appl. Phys. Lett. 72, 3172 (1998).

${ }^{11}$ J. P. Silveira and F. Briones, J. Cryst. Growth 201, 113 (1999).

${ }^{12}$ J. A. Floro, E. Chason, S. R. Lee, R. D. Twesten, R. Q. Hwang, and L. B. Freund, J. Electron. Mater. 26, 969 (1997).

${ }^{13}$ L. B. Freund, J. A. Floro, and E. Chason, Appl. Phys. Lett. 74, 1987 (1999)

${ }^{14} 1$ equivalent ML is defined as the number of atoms per unit area needed to form an complete InAs layer pseudomorphic to GaAs, 6.26 $\times 10^{14}$ atoms $/ \mathrm{cm}^{-2}$.

${ }^{15}$ J. P. Silveira, J. M. García and F. Briones (unpublished).

${ }^{16}$ This In probably will be oxidized when exposed to air.

${ }^{17}$ T. R. Ramachandran, R. Heitz, P. Chen, and A. Madhukar, Appl. Phys. Lett. 70, 640 (1997)

${ }^{18}$ N. Moll, M. Scheffler, and E. Pehlke, Phys. Rev. B 58, 4566 (1998).

${ }^{19}$ D. Leonard, K. Pond, and P. M. Petroff, World Sci. 51, 648 (1995).

${ }^{20}$ B. W. Wessels, J. Vac. Sci. Technol. B 15, 1056 (1997). 